\title{
Síntese de observadores PI baseada em otimização evolutiva multiobjetivo $\mathcal{H}_{\infty} / \mathcal{H}_{2}$ *
}

\author{
Flávia Cordeiro Martins*, Danielle Silva Gontijo*, \\ Eduardo Nunes Gonçalves** \\ *Programa de Pós-graduação em Engenharia Elétrica \\ UFSJ/CEFET-MG \\ **Departamento de Engenharia Elétrica - CEFET-MG, Av. Amazonas \\ 7675 - Belo Horizonte, $M G$ \\ (e-mail: fmartins.engeletrica@gmail.com,daniellegontijo@ymail.com, \\ eduardong@cefetmg.br)
}

\begin{abstract}
This paper deals with the PI observer synthesis applied to state-feedback control. The objectives are to propose a synthesis formulation and to compare the PI observer with the classical Luenberger observer. We propose a $\mathcal{H}_{\infty} / \mathcal{H}_{2}$ multiobjective optimization problem formulation to compute an observer that achieves a trade-off between minimization of the error due to disturbances, that requires higher observer gains, and minimization of the variance of the estimation error due to measurement noises, that requires lower observer gains. Minimizing the variance of the estimation error results in the corresponding minimization of the variance of the control action that is based on the estimated state variables. We consider a case study to analyze if the PI observer outperforms the classical Luenberger observer for state estimation applied to state-feedback control. The results showed that it is possible to design a PI observer with the same performance as the Luenberger observer but with smaller gains, which results in better attenuation of measurement noise.

Resumo: Neste trabalho é proposto um método de síntese de observador Proporcional Integral (PI) aplicado ao controle por realimentação de estados. O objetivo é propor uma formulação de síntese e comparar o observador PI com o observador clássico de Luenberger. Um problema de otimização multiobjetivo $\mathcal{H}_{\infty} / \mathcal{H}_{2}$ é formulado para projetar um observador que alcance um compromisso entre minimização do erro na presença de perturbações de entrada, o que exige maiores ganhos do observador e a minimização da variância do erro de estimação devido a ruídos de medição, que requer ganhos mais baixos do observador. A minimização da variação do erro de estimação resulta na correspondente minimização da variação da ação de controle com base nas variáveis de estado estimadas. Consideramos um estudo de caso para analisar se o observador PI supera o observador clássico de Luenberger quando a estimação de estados é aplicada ao controle por realimentação de estados. Os resultados mostraram que é possível projetar um observador PI com mesmo desempenho do observador de Luenberger porém com ganhos menores, o que resulta em melhor atenuação dos ruídos de medição.
\end{abstract}

Keywords: PI observer, $\mathcal{H}_{\infty} / \mathcal{H}_{2}$ performance, multiobjective evolutionary optimization. Palavras-chaves: Observador PI, Desempenho $\mathcal{H}_{\infty} / \mathcal{H}_{2}$, otimização evolutiva multiobjetivo.

\section{INTRODUÇÃO}

Um observador é um sistema que fornece uma estimativa dos estados ou de entradas desconhecidas, como perturbações, a partir de medições da entrada e saída do sistema real. No projeto de controle por realimentação de estados, pode ser necessário aplicar o observador, pois nem sempre é possível medir todos os estados. O observador clássico de Luenberger baseia-se no princípio da construção de um modelo do sistema real, a partir das mesmas entradas do sistema original, e utiliza o vetor de estado do modelo para estimar o vetor de estado real (Luenberger, 1964).

* Os autores agradecem os apoios das agências CAPES, CNPq, e FAPEMIG, [APQ-02943-15] - Edital 01/2015 Demanda Universal.
A principal desvantagem do observador clássico é apresentar erros de estimação na presença de perturbações não mensuráveis. Para reduzir o erro de estimação, calcula-se o erro entre a saída medida do sistema real e a saída do modelo e aplica-se uma matriz de ganhos para correção dos estados estimados. Entretanto, a malha de realimentação proporcional reduz, mas não elimina o erro de estimação de estado na presença de distúrbios. Uma possibilidade de aperfeiçoar o observador clássico é considerar um observador Proporcional Integral (PI) como apresentado na Figura 1 (Wojciechowski, 1978). O observador clássico de Luenberger possui apenas a matriz de ganho $L_{1}$, enquanto o observador PI inclui a malha de realimentação adicional com o bloco integrador e as matrizes de ganho $L_{2}$ e $L_{3}$. A 
ação integral é aplicada como combinação linear dos erros de estimação da saída.

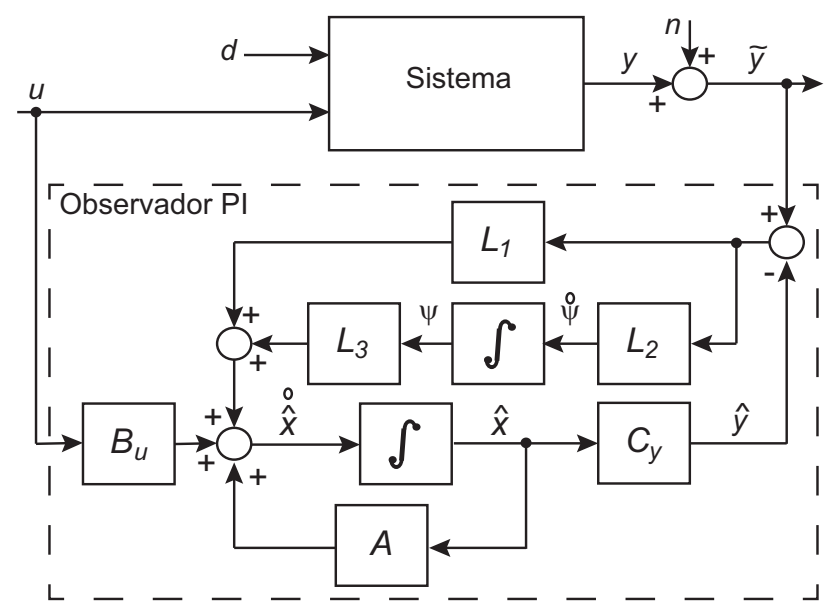

Figura 1. Observador Proporcional Integral.

Estruturas e técnicas de síntese para observadores PI foram propostos e aplicadas com sucesso em diferentes cenários. Uma abordagem para projeto de observadores PI em sistemas lineares multivariáveis baseada na especificação de autovalores foi proposta em Duan et al. (2001). Diferentes métodos e estruturas de projeto, incluindo observadores PI de alto ganho escalonado, observador PI avançado e observador PI modificado avançado foram introduzidos para melhorar a robustez da estimação e o desempenho do observador PI original (Bakhshande and Söffker, 2015). O desempenho $\mathcal{H}_{\infty}$ e a formulação LMI também são considerados em projetos de controle chaveado robustos, utilizando a técnica de controle baseado no observador PI para sistemas chaveados sujeito a incertezas e perturbações externas (Menhour et al., 2017). A síntese de observadores PI baseado no desempenho misto $\mathcal{H}_{\infty} / \mathcal{H}_{2}$ e formulação LMI, também tem sido proposta para estimação de perturbações e torques de partidas em sistema de direção elétrica (Yamamoto et al., 2015). O observador PI foi aplicado no controle de corrente de acionamentos de máquinas síncronas de ímã permanente usando um controlador proporcional puro no caminho principal e compensando a perturbação com o termo integral do observador PI (De Soricellis et al., 2018).

A contribuição deste trabalho é apresentar uma formulação para a síntese de observadores PI a ser aplicada ao controle por realimentação de estados baseado em observador. Semelhante ao caso da estimação de perturbações em Yamamoto et al. (2015), também propomos uma formulação de síntese baseada no problema de otimização multiobjetivo $\mathcal{H}_{\infty} / \mathcal{H}_{2}$. Diferente de Yamamoto et al. (2015), resolvemos o problema de controle por meio de um algoritmo evolucionário multiobjetivo que fornece um conjunto de soluções eficientes com diferentes compromissos entre as funções objetivo.

As especificações de projeto são quantificadas em termos das normas $\mathcal{H}_{\infty}$ e $\mathcal{H}_{2}$. A norma $\mathcal{H}_{\infty}$ representa a relação entre a energia do sinal de saída e a energia do sinal de entrada, ou seja quanto menor a norma $\mathcal{H}_{\infty}$, maior é a rejeição de perturbação na forma de energia limitada. Já a norma $\mathcal{H}_{2}$, é utilizada para tratar a rejeição de distúrbios estocásticos, tais como ruídos de medição ou perturbações aleatórias. Desta forma, minimizar a norma $\mathcal{H}_{2}$ trata-se da minimização da relação da variância do sinal de saída com a variância do sinal de entrada (Zhou and Doyle, 1998).

É apresentado um estudo de caso para comparar os observadores clássicos de Luenberger e PI. Neste estudo de caso, foi aplicada a formulação proposta para calcular um conjunto eficiente de observadores clássicos de Luenberger e PI para implementar o controle por realimentação de estados, obtendo diferentes compromissos entre a minimização do erro de estimação de estado devido a perturbações e ruídos de medição. O objetivo do estudo de caso é verificar se o observador PI pode superar o observador de Luenberger e também a influência da escolha das dimensões das matrizes $L_{2}$ e $L_{3}$ (número de integradores).

A notação padrão e compacta utilizada neste artigo:

$$
G(s)=\left[\begin{array}{l|l}
A & B \\
\hline C & D
\end{array}\right]
$$

é aplicada para denotar a função de transferência $G(s)=$ $C(s I-A)^{-1} B+D$. A matriz $I$ é a matriz identidade com dimensão apropriada.

\section{FORMULAÇÃO DO PROBLEMA}

Considere um sistema contínuo linear invariante no tempo representado em espaço de estados:

$$
\begin{aligned}
& \dot{x}(t)=A x(t)+B_{u} u(t)+B_{d} d(t), \\
& y(t)=C_{y} x(t),
\end{aligned}
$$

sendo $x(t) \in \mathbb{R}^{n}$ o vetor de estados, $u(t) \in \mathbb{R}^{p}$ o vetor de entrada, $d(t) \in \mathbb{R}^{n_{d}}$ o vetor de perturbação e $y(t) \in$ $\mathbb{R}^{q}$ o vetor de saída do sistema. Considere o observador proporcional integral (PI) apresentado na Figura 1. A diferença entre o observador Luenberger tradicional e o observador PI é incluir os blocos de matrizes de ganhos $L_{2}$, $L_{3}$ e o bloco integral em paralelo com o bloco da matriz de ganhos $L_{1}$. Definindo o erro de estimação $e(t) \triangleq x(t)-$ $\hat{x}(y), \hat{x}(t)$ o vetor de estados estimados, a derivada do erro de estimação é dada por:

$$
\dot{e}(t)=\left(A-L_{1} C_{y}\right) e(t)-L_{3} \psi(t)-L_{1} n(t)+B_{d} d(t)
$$

sendo $n(t) \in \mathbb{R}^{q}$ o vetor de ruído de medição. Considerando a configuração de controle apresentada na Figura 2, define-se $\dot{z} \triangleq r(t)-[y(t)+n(t)]$ igual à integral do erro de rastreamento, com $r(t)$ o vetor de sinal de referência, a ação de controle é:

$$
u(t)=K_{x} \hat{x}(t)+K_{i} z(t)
$$

O modelo em espaço de estados do controle por realimentação de estados, com ação integral, baseado no observador PI é:

$$
\begin{aligned}
{\left[\begin{array}{c}
\dot{x} \\
\dot{z} \\
\dot{e} \\
\dot{\psi}
\end{array}\right]=} & {\left[\begin{array}{cccc}
A+B_{u} K_{x} & B_{u} K_{i} & -B_{u} K_{x} & 0 \\
-C_{y} & 0 & 0 & 0 \\
0 & 0 & A-L_{1} C_{y} & -L_{3} \\
0 & 0 & L_{2} C_{y} & 0
\end{array}\right]\left[\begin{array}{c}
x \\
z \\
e \\
\psi
\end{array}\right] } \\
+ & {\left[\begin{array}{ccc}
0 & B_{d} & 0 \\
I & 0 & -I \\
0 & B_{d} & -L_{1} \\
0 & 0 & L_{2}
\end{array}\right]\left[\begin{array}{l}
r \\
d \\
n
\end{array}\right] }
\end{aligned}
$$

Observando a Equação (4), podemos notar que o controle por realimentação de estados baseado no observador PI também apresenta o princípio da separação como é o caso 


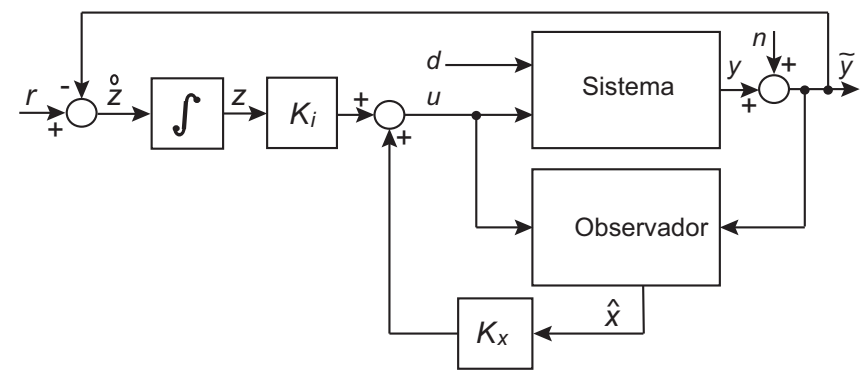

Figura 2. Realimentação de estados com ação integral baseado no observador PI.

do observador de Luenberger. Isso significa que o ganho de realimentação de estados $K \triangleq\left[\begin{array}{ll}K_{x} & K_{i}\end{array}\right] \in \mathbb{R}^{p \times(n+q)}$ pode ser calculado independentemente das matrizes de ganho do observador PI, $L_{1} \in \mathbb{R}^{n \times q}, L_{2} \in \mathbb{R}^{r \times q}$, e $L_{3} \in \mathbb{R}^{n \times r}$, onde $r$ é a dimensão arbitrária de $\psi \in \mathbb{R}^{r}$. As escolhas possíveis são $r=1$ ou $r=q$, mesma dimensão do vetor da saída do sistema.

Definindo:

$$
A_{0} \triangleq\left[\begin{array}{cc}
A-L_{1} C y & -L_{3} \\
L_{2} C_{y} & 0
\end{array}\right], \bar{B}_{d} \triangleq\left[\begin{array}{c}
B_{d} \\
0
\end{array}\right], \bar{L} \triangleq\left[\begin{array}{c}
-L_{1} \\
L_{2}
\end{array}\right],
$$

então as matrizes de transferência relacionando os distúrbios e erro de estimação, $T_{e d}(s)$, ruídos de medição e erro de estimação, $T_{e n}(s)$, são:

$$
T_{e d}(s)=\left[\begin{array}{c|c}
A_{0} & \bar{B}_{d} \\
\hline C_{0} & 0
\end{array}\right], T_{e n}(s)=\left[\begin{array}{c|c}
A_{0} & \bar{L} \\
\hline C_{0} & 0
\end{array}\right],
$$

sendo $C_{0}=\left[\begin{array}{ll}I_{q \times q} & 0_{q \times r}\end{array}\right]$.

O problema de síntese do observador PI é apresentado como: para um dado $r$, calcula-se as matrizes $L_{1} \in$ $\mathbb{R}^{n \times q}, L_{2} \in \mathbb{R}^{r \times q}$, e $L_{3} \in \mathbb{R}^{n \times r}$ que minimizam o efeito das perturbações, $d(t)$, e os ruídos de medição, $n(t)$, sobre o erro de estimação de estado, $e(t)$. Estes dois objetivos são conflitantes, uma vez que um pequeno erro na presença de perturbação requer maiores ganhos do observador, enquanto a atenuação do ruído requer menores ganhos do observador. Formulou-se o problema de síntese do observador PI como um problema de otimização multiobjetivo $\mathcal{H}_{\infty} / \mathcal{H}_{2}$ :

$$
\begin{gathered}
\theta^{*}=\arg \min _{\theta}\left[\begin{array}{c}
\left\|T_{e d}(s, \theta)\right\|_{\infty} \\
\left\|T_{e n}(s, \theta)\right\|_{2}
\end{array}\right] \\
\text { sujeito a: } \\
\eta\left(A_{0}\right)<0 \\
\left\|T_{e n}(s, \theta)\right\|_{2} \leq \epsilon
\end{gathered}
$$

sendo $\theta \in \mathbb{R}^{v}, v=n \times q+r \times q+n \times r$, é o vetor das variáveis de otimização que são os elementos das matrizes $L_{1}, L_{2}$, e $L_{3}$, e $\eta\left(A_{0}\right)=\max _{i}\left\{\Re\left(\lambda_{i}\left(A_{0}\right)\right)\right\}$ é sendo $\Re(\lambda)$ a parte real $\lambda \in \mathbb{C}$ e $\left.\lambda_{i}\left(A_{0}\right)\right)$ o $i$-ésimo autovalor da matriz $A_{0}$. A segunda restrição é incluída para reduzir o espalhamento das soluções do Pareto.

\section{OTIMIZAÇÃO EVOLUCIONÁRIA MULTIOBJETIVO}

Os problemas multiobjetivo possuem um conjunto de soluções eficientes ou não dominadas ou o conjunto Pareto. Uma solução $\theta^{*}$ do problema multiobjetivo é eficiente se não houver outra melhor solução $\theta$ tal que $f(\theta) \leq f\left(\theta^{*}\right)$ e $f(\theta) \neq f\left(\theta^{*}\right)$. Para os vetores $X=\left[x_{1} \ldots x_{k}\right]^{T}$ e $Y=$ $\left[y_{1} \ldots y_{k}\right]^{T}, X \leq Y$ se $x_{i} \leq y_{i}$ para $i=1, \ldots, k$, e $X \neq Y$ se $\exists i \mid x_{i} \neq y_{i}$. O problema de otimização multiobjetivo é encontrar um subconjunto do conjunto de Pareto:

$$
\Theta^{*}=\left\{\theta^{*} \in \mathcal{F}_{\theta}: \nexists \theta \theta \in \mathcal{F}_{\theta} \mid f(\theta) \leq f\left(\theta^{*}\right) ; f(\theta) \neq f\left(\theta^{*}\right)\right\}
$$

sendo $\mathcal{F}_{\theta}$ o conjunto de soluções factíveis.

Existem vários algoritmos de otimização multiobjetivo para gerar um subconjunto candidato do conjunto Pareto, por exemplo, o Algoritmo Genético de Ordenação Não Dominado (NSGA-II) (Deb et al., 2002), Evolução Diferencial para Otimização Multiobjetivo (DEMO) (Robič and Filipič, 2005) ou Algoritmo Evolutivo Multiobjetivo Baseado na Decomposição (MOEA/D) (Zhang and Li, 2007). Neste trabalho o algoritmo DEMO foi aplicado para resolver o problema multiobjetivo (7) considerando experiências anteriores com os três métodos citados.

O algoritmo de Evolução Diferencial (DE) para problemas escalares, foi introduzido por R. Storn e K. V. Price em 1995, com a inclusão de operadores computacionais semelhantes aos empregados por algoritmos evolucionários padrão (Storn and Price, 1997; Das et al., 2016).

Seja $\mathcal{U}_{(a, b)} \in \mathbb{R}$ um número pseudoaleatório uniformemente distribuído no intervalo aberto $(a, b) ; \mathcal{I}_{(m)} \in \mathbb{Z}^{+}$é um valor inteiro pseudo-aleatório extraído da distribuição uniforme discreta de $1, \ldots, m$, sendo $m$ o limite superior; $\mathbf{x} \in \mathbb{R}^{n}$ é o vetor das variáveis de otimização; e $N$ é o número de indivíduos (soluções candidatas) da população. A população na $k$-ésima iteração representada por $X_{k}=\left\{\mathbf{x}_{k, i} ; i=1, \ldots, N\right\}$, em que a $i$-ésima solução é:

$$
\mathbf{x}_{k, i}=\left[\theta_{k, i, 1} \ldots \theta_{k, i, n}\right]^{T} .
$$

As operações do DE são descritas na sequência.

O método DE começa com uma população inicial de soluções candidatas distribuídos aleatoriamente e uniformemente dentro do espaço de busca inicial restrito pelos limites mínimo e máximo pré-definidos de cada variável, $\theta_{1, i, j} \in\left[\underline{m}_{j}, \bar{m}_{j}\right]$ :

$$
\theta_{1, i, j}=\mathcal{U}_{\left(\underline{m}_{j}, \bar{m}_{j}\right)}
$$

$i=1, \ldots, N, j=1, \ldots, n$.

Considere os índices $r_{1} \neq r_{2} \neq r_{3} \neq i$ gerados como $r_{j}=\mathcal{I}_{(N)}, j=1, \ldots, 3$. A $i$-ésima solução mutante é calculada com base na operação diferencial de mutação:

$$
\mathbf{v}_{k, i}=\mathbf{x}_{k, r_{1}}+F_{i} \cdot\left(\mathbf{x}_{k, r_{2}}-\mathbf{x}_{k, r_{3}}\right),
$$

$i=1, \ldots, N$, sendo que $F_{i}$ é um fator de escala aplicado sobre o vetor de diferença. Neste trabalho, adotou-se $F_{i}=$ $\mathcal{U}_{(0.5,1)}$ para cada vetor de diferença.

A recombinação entre os indivíduos da $k$-ésima população, $X_{k}$, e a população mutante, $V_{k}$, produz a população auxiliar, $U_{k}$ :

$$
u_{k, i, j}=\left\{\begin{array}{l}
v_{k, i, j}, \text { se } \mathcal{U}_{(0,1)} \leq C_{r} \text { ou } j=\delta_{i} \\
\theta_{k, i, j}, \text { caso contrário. }
\end{array},\right.
$$

$i=1, \ldots, N, j=1, \ldots, n$, onde $\delta_{i}=\mathcal{I}_{(n)}$ garante $\mathbf{u}_{k, i} \neq \mathbf{x}_{k, i}$ e $C_{r} \in[0,1]$ é a taxa de cruzamento. Neste trabalho, considerou-se $C_{r}=0.9$.

A próxima etapa do algoritmo é a operação de seleção para determinar se o indivíduo alvo, $\mathbf{x}_{k, i}$, ou o vetor de teste, $\mathbf{u}_{k, i}$, sobrevive à próxima geração. Nesta etapa, o algoritmo multiobjetivo DEMO difere do DE original:

$$
\mathbf{x}_{k+1, i}=\left\{\begin{array}{l}
\mathbf{u}_{k, i}, \text { se } f\left(\mathbf{u}_{k, i}\right) \leq f\left(\mathbf{x}_{k, i}\right) \text { e } f\left(\mathbf{u}_{k, i}\right) \neq f\left(\mathbf{x}_{k, i}\right) \\
\mathbf{x}_{k, i}, \text { caso contrário. }
\end{array}\right.
$$

$i=1, \ldots, N$. Se $\mathbf{x}_{k, i}$ não domina $\mathbf{u}_{k, i}$, então $\mathbf{u}_{k, i}$, também é incluído no conjunto $X_{k+1}$, aumentando o tamanho da população. 
Após a operação de seleção, se o número de soluções de $X_{k+1}$ for maior que $N$, para selecionar as melhores $N$ soluções de $X_{k+1}$, o algoritmo DEMO aplica as mesmas técnicas de seleção do algoritmo NSGA-II com classificação por índice de fronteiras com desempate pelo índice de aglomeração, para alcançar uma boa dispersão ou diversidade de soluções (Deb et al., 2002).

O algoritmo DE pode ser aplicado a problemas de otimização sem restrições. Para incluir as duas restrições, aplicouse o método de funções penalidade. O algoritmo DEMO foi aplicado para minimizar a função objetiva modificada:

$$
f_{o}(\theta)= \begin{cases}{\left[\begin{array}{l}
10^{12}+\eta\left(A_{0}\right) \\
10^{12}+\eta\left(A_{0}\right)
\end{array}\right],} & \text { se } \eta\left(A_{0}\right) \geq 0, \\
{\left[\begin{array}{l}
10^{6}+\left\|T_{e n}(s, \theta)\right\|_{2}-\epsilon \\
10^{6}+\left\|T_{e n}(s, \theta)\right\|_{2}-\epsilon
\end{array}\right],} & \text { se }\left\|T_{e n}(s, \theta)\right\|_{2}>\epsilon \\
{\left[\begin{array}{cc}
\left\|T_{e d}(s, \theta)\right\|_{\infty} \\
\left\|T_{e n}(s, \theta)\right\|_{2}
\end{array}\right],} & \text { caso contrário. }\end{cases}
$$

Otimizando $f_{o}(\theta)$, as soluções factíveis vão dominar as soluções que violam as restrições.

\section{RESULTADOS COMPUTACIONAIS}

Para ilustrar a aplicação do procedimento de síntese proposto, considere um exemplo ilustrativo de um processo de controle de nível de água com quatro tanques (Johansson, 2000), apresentado na Figura 3. Esse sistema pode ser representado pelo modelo em espaço de estados linearizado:

$$
\begin{aligned}
\dot{x} & =\left[\begin{array}{cccc}
-\frac{1}{T_{1}} & 0 & \frac{A_{3}}{A_{1} T_{3}} & 0 \\
0 & -\frac{1}{T_{2}} & 0 & \frac{A_{4}}{A_{2} T_{4}} \\
0 & 0 & -\frac{1}{T_{3}} & 0 \\
0 & 0 & 0 & -\frac{1}{T_{4}}
\end{array}\right] x \\
+ & {\left[\begin{array}{cc}
\frac{\gamma_{1} k_{1}}{A_{1}} & 0 \\
0 & \frac{\gamma_{2} k_{2}}{A_{2}} \\
0 & \frac{\left(1-\gamma_{2}\right) k_{2}}{A_{3}} \\
\frac{\left(1-\gamma_{1}\right) k_{1}}{A_{4}} & 0
\end{array}\right] u+\left[\begin{array}{cc}
\frac{1}{A_{1}} & 0 \\
0 & \frac{1}{A_{2}} \\
0 & 0 \\
0 & 0
\end{array}\right] d, } \\
y & =\left[\begin{array}{ccc}
k_{c} & 0 & 0 \\
0 & k_{c} & 0
\end{array}\right] x,
\end{aligned}
$$

sendo as variáveis de estado os quatro desvios de nível do tanque, os sinais de controle são os dois desvios de tensão da bomba, todos em relação ao ponto de operação. O modelo original foi modificado para incluir duas perturbações, sendo estas, vazões nas entrada dos tanques 1 e 2 . O modelo linearizado dos quatro tanques no ponto de operação de fase mínima tem os seguintes valores de parâmetros (Johansson, 2000): seções transversais do tanque $A_{1}=A_{3}=28 \mathrm{~cm}^{2}, A_{2}=A_{4}=32 \mathrm{~cm}^{2}$; ganho do sensor $k_{c}=0.50 \mathrm{~V} / \mathrm{cm} ;$ ganhos das bombas $\left[k_{1}, k_{2}\right]=[3.33,3.35]$, válvula de três vias $\left[\gamma_{1}, \gamma_{2}\right]=[0.70,0.60]$, e constantes linearizadas $\left[T_{1}, T_{2}, T_{3}, T_{4}\right]=[62,90,23,30]$. As matrizes de ganho ótimas da realimentação de estados baseado no projeto do Regulador Linear-Quadrático (LQR) foram calculadas:

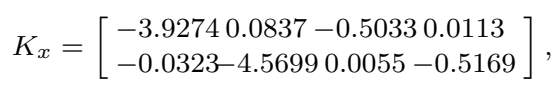

$$
\begin{aligned}
& K_{i}=\left[\begin{array}{cc}
1.4141 & -0.0187 \\
0.0187 & 1.4141
\end{array}\right] .
\end{aligned}
$$

O ganho $K_{i}$ corresponde a duas variáveis de estado adicionais iguais as integrais dos erros entre os níveis dos

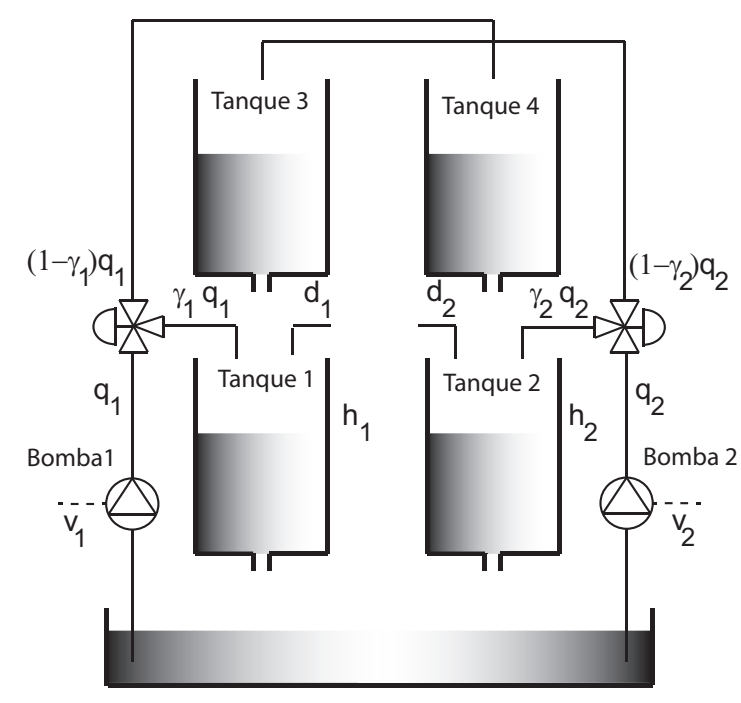

Figura 3. Processo de quatro tanques para estimação e controle dos níveis de água.

tanques 1 e 2 no ponto de equilíbrio. O algoritmo DEMO foi aplicado com $N=50$ e número de gerações $N_{g}=1000$, para calcular o conjunto de candidatos Pareto adotando a $\left\|T_{e n}(s)\right\|_{2} \leq 25$ (para excluir as soluções com altos ganhos). As curvas candidatas de Pareto são apresentadas na Figura 4 para os observadores de Luenberger e PI, para $r=1$ e $r=2$.

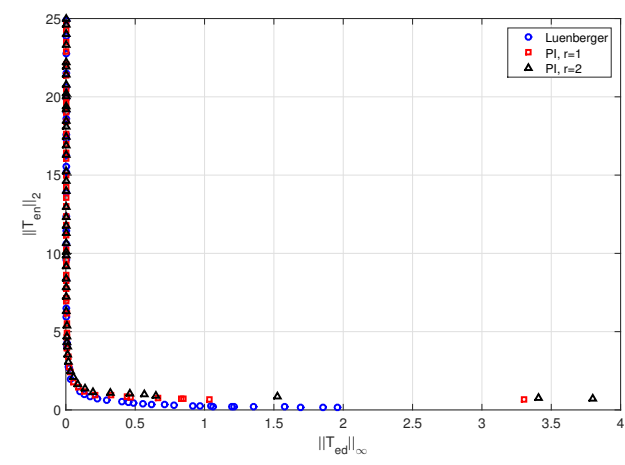

Figura 4. Curva de candidatos Pareto.

Seja $\mathbf{1}(t-\tau)$ a função degrau unitário com $\mathbf{1}(t)=0$ para $t<\tau$ e $\mathbf{1}(t)=1$ para $t \geq \tau$, e $\rho(t)$ ser uma função que gera valores pseudo-aleatórios com distribuição normal padrão, média 0 e variância 1 . Analisou-se o comportamento dos observadores selecionados para os seguintes sinais: $d_{1}(t)=$ $4 \times \mathbf{1}(t), d_{2}(t)=4 \times \mathbf{1}(t-300)$, e $n_{i}=0.01 \rho(t), i=$ 1,2 . Os sinais de referência estão em Volts enquanto as variáveis de estado estão em centímetros relacionadas por $k_{c}=0.5 \mathrm{~V} / \mathrm{cm}$. Considerou-se os sinais de perturbação um degrau, para analisar os erros de estimação em estado estacionário. Apesar do sinal degrau não ser de energia limitada, ele foi considerado para verificar a capacidade do observador em acompanhar o desvio em relação ao ponto de operação. O resultado da simulação obtida após 30 testes apresentou o mesmo erro de estimação em estado estacionário na presença de perturbações com o observador Luenberger e o PI.

Considerou-se a integral do erro ao quadrado como índice de desempenho para comparar os diferentes observadores: 


$$
\begin{gathered}
I S E_{i} \triangleq \int_{0}^{\infty}\left[\left(r_{i}(t)-y_{i}(t)\right]^{2} d t,\right. \\
I S E_{t} \triangleq \sum_{i=1}^{q} I S E_{i} .
\end{gathered}
$$

A título de comparação, analisou-se o primeiro observador de cada tipo que atinge o $I S E_{t} \leq 0.55$ (escolha baseada nos resultados da simulação) quando o sistema é submetido às perturbações sem ruído. A matriz de ganho do observador Luenberger selecionada, com 8 variáveis de otimização, é:

$$
L=\left[\begin{array}{cc}
211.8528 & -0.9450 \\
-0.0362 & 183.0980 \\
0.0142 & -0.0202 \\
-0.0170 & -0.0129
\end{array}\right],
$$

as matrizes de ganho do observador PI, para $r=1$, com 14 variáveis de otimização, são:

$$
\begin{gathered}
L_{1}=\left[\begin{array}{cc}
97.7301 & 0.7838 \\
1.6149 & 81.0385 \\
-0.0645 & 1.2466 \\
0.0088 & -0.4648
\end{array}\right], \\
L_{2}=\left[\begin{array}{ll}
-0.2275 & 5.7984
\end{array}\right], L_{3}=\left[\begin{array}{c}
1.6295 \\
35.3529 \\
-0.0040 \\
-0.0198
\end{array}\right],
\end{gathered}
$$

e as matrizes de ganho do observador PI, para $r=2$, com $L_{2}$ fixa como matriz diagonal, com 18 variáveis de otimização, são:

$$
\begin{gathered}
L_{1}=\left[\begin{array}{cc}
19.9697 & -0.4650 \\
-0.2508 & 17.7146 \\
0.0683 & 0.2370 \\
-0.1311 & -0.0835
\end{array}\right], L_{2}=\left[\begin{array}{cc}
-0.0370 & 0 \\
0 & 0.0754
\end{array}\right], \\
L_{3}=\left[\begin{array}{cc}
-229.1006 & 5.3732 \\
-9.5244 & 185.5112 \\
-0.1001 & -0.2191 \\
-0.1021 & 0.0083
\end{array}\right]
\end{gathered}
$$

Os primeiros 50s das respostas transitórias das variáveis de estado reais e estimadas, são semelhantes para os três observadores, sem ruídos de medição, com $I S E_{t} \leq$ 0.55, como apresentados na Figura 5. É possível ver na Figura 5 que a perturbação é rejeitada e a diferença entre as variáveis reais e estimadas não são significativas (elas tendem a zero). Para alcançar um erro de estimação menor na presença de perturbações, é necessário um alto ganho do observador, o que pode ser um problema na presença de ruídos de medição. As respostas transitórias das entradas de tensão da bomba e dos níveis simulados e estimados de $h_{1}(t)$ e $h_{2}(t)$, considerando os ruídos de medição, são apresentadas nas Figuras 6 e 7 , para o observador de Luenberger, e nas Figuras 8 e 9 para o observador PI, com $r=2$ (resultados intermediários para $r=1$ ).

Tabela 1. Desempenho dos observadores na presença de ruídos e perturbações

\begin{tabular}{cccc} 
& $I S E_{t}$ & $\operatorname{Var}\left(u_{1}\right)$ & $\operatorname{Var}\left(u_{2}\right)$ \\
\hline Luenberger & 0.5494 & $0.1946 \times 10^{-3}$ & $0.4856 \times 10^{-3}$ \\
PI, $r=1$ & 0.5495 & $0.0634 \times 10^{-3}$ & $0.2364 \times 10^{-3}$ \\
PI, $r=2$ & 0.5495 & $0.0592 \times 10^{-4}$ & $0.2963 \times 10^{-4}$ \\
\hline
\end{tabular}

A Tabela 1 lista o $I S E_{t}$ e a variância de cada entrada nas últimas 200 amostras, para os três observadores. Todos os observadores apresentam $I S E_{t} \leq 0.55$, mas as variâncias da estimação de $h_{1}(t)$ e $h_{2}(t)$ e, consequentemente, a variância das tensões da bomba, no observador de Luenberger, é maior que no observador PI com $r=1$ e maior que no observador PI com $r=2$.

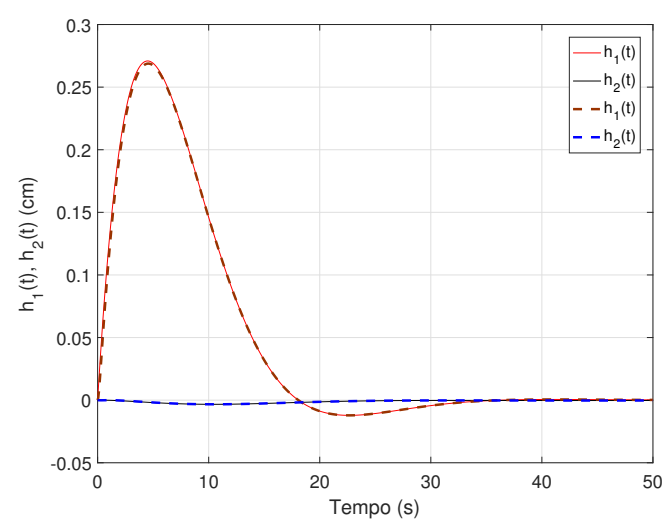

Figura 5. Respostas transitórias parciais dos desvios de nível simulado (sólida) e estimados (tracejada) dos tanques 1 e 2 sem ruídos de medicão.

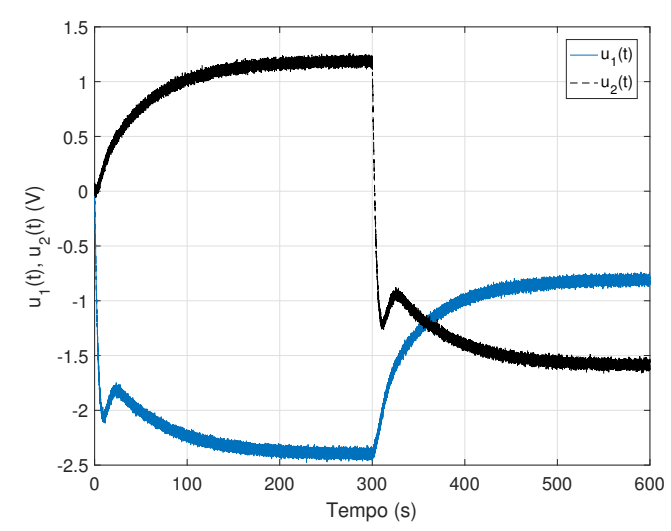

Figura 6. Respostas transitórias das tensões das bombas para o observador de Luenberger.

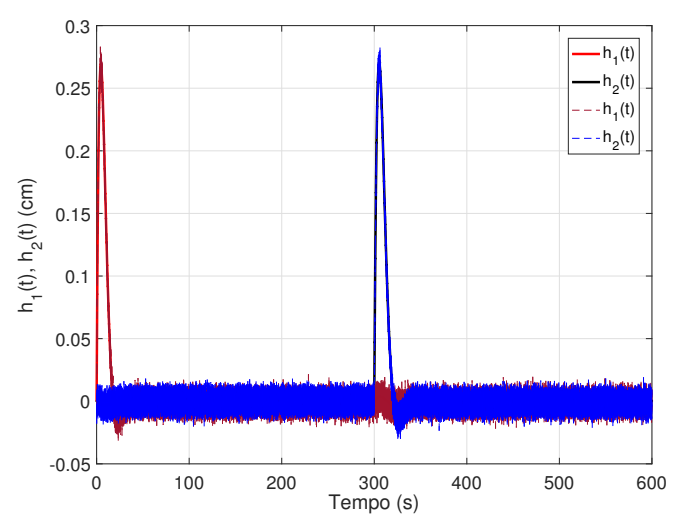

Figura 7. Respostas transitórias do sistema (sólida) e desvios de nível estimados (tracejadas), $h_{1}(t)$ e $h_{2}(t)$ para o observador de Luenberger.

A variação da estimação de $h_{1}(t)$ e $h_{2}(t)$ oculta as variáveis de estado simuladas que apresentam menor variação. Os erros de estimação para $h_{3}(t)$ e $h_{4}(t)$ são insignificantes em todos os casos porque não são influenciados diretamente pelos distúrbios. Conclui-se que, para este estudo de caso, 
na presença de ruídos de medição, o observador PI com $r=2$ supera os outros dois observadores.

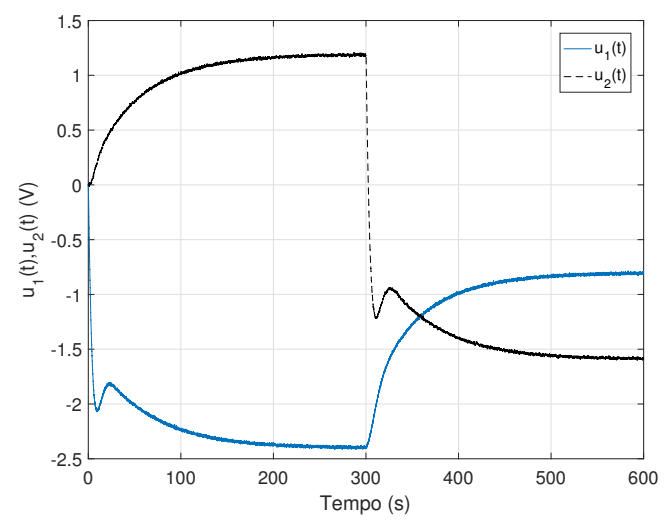

Figura 8. Respostas transitórias das tensões das bombas para o observador PI com $r=2$.

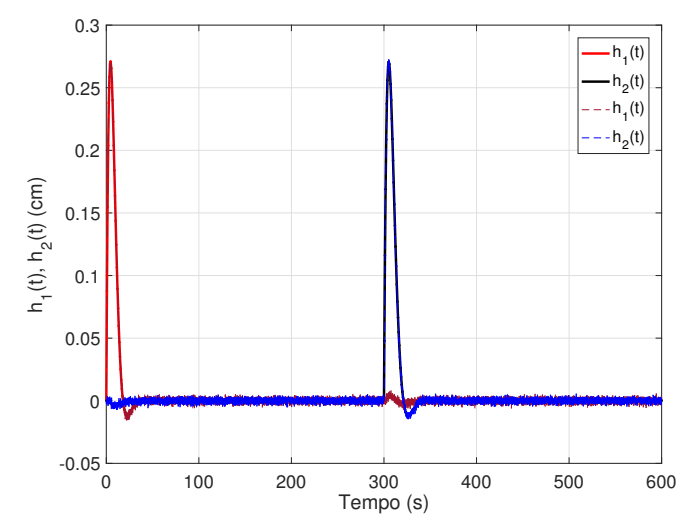

Figura 9. Respostas transitórias do sistema (sólida) e desvios de nível estimados (tracejada), $h_{1}(t)$ e $h_{2}(t)$ do observador PI com $r=2$.

Vale ressaltar que, para $r=2$, o erro $\tilde{y}-\hat{y}$ está relacionado ao erro de estimação de estados de $h_{1}(t)$ e $h_{2}(t)$, o que garante que o valor médio desses erros tenda a zero em estado estacionário. Havia a possibilidade de analisar os observadores com valores semelhantes de funções objetivo (localização mais próxima nas curvas candidatas de Pareto, Figura 4). No entanto, para valores objetivos similares ao observador PI $(r=2)$ apresentado, os observadores de Luenberger e PI $(r=1)$ apresentam menores ganhos, em consequência, menores variações de entrada, mas maiores erros de estimação em estado estacionário para as quatro variáveis de estados e maiores $I S E_{t}$.

\section{CONCLUSÃO}

Foi proposto uma formulação para a síntese de observadores PI aplicada ao controle por realimentação de estados. A formulação de síntese é baseada no problema de otimização multiobjetivo $\mathcal{H}_{\infty} / \mathcal{H}_{2}$, que pode ser resolvido por meio de algoritmos evolutivos multiobjetivo. Aplicou-se a abordagem proposta para calcular um conjunto eficiente de observadores PI e de Luenberger para implementar um controle por realimentação de estados, obtendo diferentes compromissos entre a minimização de perturbações e os ruídos de medição sobre os erro de estimação de estado. Consideramos um estudo de caso para comparar as duas estruturas de observadores. Os resultados da simulação alcançados demonstraram que o observador PI supera o observador de Luenberger ao comparar o desempenho na atenuação de ruídos. Ambos os observadores apresentam erros de estimação em estado estacionário quando o sistema é submetido a perturbações constantes que podem ser reduzidas com ganhos elevados do observador. Para o estudo de caso considerado, considerar dois integradores em vez de um no observador PI melhorou o desempenho.

\section{REFERÊNCIAS}

Bakhshande, F. and Söffker, D. (2015). Proportionalintegral-observer: A brief survey with special attention to the actual methods using ACC benchmark. IFACPapersOnLine, 48(1), 532-537.

Das, S., Mullick, S.S., and Suganthan, P.N. (2016). Recent advances in differential evolution - an updated survey. Swarm and Evolutionary Computation, 1, 1-30.

De Soricellis, M., Da Ru, D., and Bolognani, S. (2018). A robust current control based on proportional-integral observers for permanent magnet synchronous machines. IEEE Transactions on Industry Applications, 54(2), 1437-1447.

Deb, K., Pratap, A., Agarwal, S., and Meyarivan, T. (2002). A fast and elitist multiobjective genetic algorithm: NSGA-II. IEEE Transaction on Evolutionary Computation, 6(2), 182-182.

Duan, G., Liu, G., and Thompson, S. (2001). Eigenstructure assignment design for proportional-integral observers: continuous-time case. IEE Proceedings-Control Theory and Applications, 148(3), 263-267.

Johansson, K.H. (2000). The quadruple-tank process: A multivariable laboratory process with an adjustable zero. IEEE Transactions on Control Systems Technology, 8(3), 456-465.

Luenberger, D.G. (1964). Observing the state of a linear system. IEEE transactions on military electronics, 8(2), 74-80.

Menhour, L., Koenig, D., and d'Andra Novel, B. (2017). Robust switched $\mathcal{H}_{\infty}$ PI observer-based controller: Vehicle dynamics application. IFAC-PapersOnLine, 50(1), 10377-10382.

Robič, T. and Filipič, B. (2005). Differential evolution for multiobjective optimization. In International Conference on Evolutionary Multi-Criterion Optimization, 520-533. Springer.

Storn, R. and Price, K. (1997). Differential evolution a simple and efficient heuristic for global optimization over continuous spaces. Journal of Global Optimization, 11(4), 341-359.

Wojciechowski, B. (1978). Analysis and synthesis of proportional-intergral observers for single-input singleoutput time-invariant continuous systems. Ph. D. dissertation, Gliwice, Poland.

Yamamoto, K., Koenig, D., Sename, O., and Moulaire, P. (2015). Driver torque estimation in electric power steering system using an $\mathcal{H}_{\infty} / \mathcal{H}_{2}$ proportional integral observer. In Decision and Control (CDC), 2015 IEEE 54th Annual Conference on, 843-848. IEEE.

Zhang, Q. and Li, H. (2007). MOEA/D: A multiobjective evolutionary algorithm based on decomposition. IEEE Transaction on Evolutionary Computation, 11(6), 712731.

Zhou, K. and Doyle, J.C. (1998). Essentials of Robust Control. Prentice-Hall. Inc., New Jersey. 